\title{
片上网络无虚通道隔离路由算法
}

\author{
谢瑞莲 ${ }^{1}$, 焦继业 ${ }^{2)}$, 刘有耀 ${ }^{2)}$ \\ ${ }^{1)}$ (西安邮电大学计算机学院 西安 710061) \\ 2) (西安邮电大学电子工程学院 西安 710061) \\ (xieruilian@xupt.edu.cn)
}

摘 要: 子网隔离方法是解决片上网络众核处理器中大量应用程序并发执行时通信数据流相互干扰的重要方法. 针 对现有子网隔离路由算法只适用于某几种特定类型的不规则子网，降低了片上系统的利用率，给出了不规则子网的 拓扑结构模型, 并结合奇偶转向模型, 提出了一种低开销的无虚通道隔离路由算法. 算法采用维序路由规则传输数 据包, 一旦遇到不规则子网边界破坏了最小路由路径时, 则改变路由策略, 采用南北边界路由和东西边界路由 2 种模 式传输数据包. 通过合成负载通信模式测试和真实应用踪迹测试, 对不同算法进行系统利用率、性能和功耗比较分 析, 结果表明所提出的隔离路由算法在不降低片上系统网络性能的情况下, 提高了系统的利用率.

关键词：片上网络; 不规则拓扑子网; 子网隔离; 隔离路由

中图法分类号: TP302

DOI: $10.3724 /$ SP.J.1089.2021.18535

\section{Isolated Routing Algorithm without Virtual Channels for Network-on-Chip}

\author{
Xie Ruilian ${ }^{1)}$, Jiao Jiye ${ }^{2)}$, and Liu Youyao ${ }^{2)}$ \\ 1) (Institute of Computer Science \& Technology, Xi' an University of Posts \& Telecommunications, Xi'an 710061) \\ ${ }^{2)}$ (Institute of Electronic Engineering, Xi' an University of Posts \& Telecommunications, Xi'an 710061)
}

\begin{abstract}
Subnet isolation method is very important to solve the traffic interference when a large number of applications execute concurrently for network-on-chip (NoC) based many-core processor. Aiming at the problem that the state-of-the-art subnet isolation routing algorithms are only suitable for some specific types of irregular topological subnets, which reduce the utilization of the on-chip system, a topology model of irregular subnets is presented, and a low-cost isolation routing algorithm (LIRA) is proposed without virtual channels based on the odd-even turn model. LIRA employs the dimensional routing algorithm to transmit packets, until the boundaries of the irregular topological subnets destroy the minimal routing path. Then, LIRA adopts two modes of north-south boundary routing and east-west boundary routing to transmit packets. Experimental results on both synthetic traffic patterns and application traces show that LIRA improves the utilization of the system without decreasing the network performance.
\end{abstract}

Key words: network-on-chip (NoC); irregular topology subnet; subnet isolation; isolation routing

半导体技术的发展和应用需求推动着处理器 设计进入(多核)甚至(众核)时代 ${ }^{[1]}$. 随着内核数量
的增加，多个应用程序会同时运行在一个众核平 台上，如计算机视觉、流媒体处理、动画、数据挖

收稿日期: 2020-08-03; 修回日期: 2021-02-27. 基金项目：国家自然科学基金(61874087，61634004). 谢瑞莲(1982一), 女，博士， 讲师, CCF 会员, 主要研究方向为片上网络拓扑结构、片上网络可靠性与性能提升; 焦继业(1977一), 男, 博士, 副教授, 硕士生导师, 主要研究方向为高性能计算单元、数模混合电路设计; 刘有耀(1975-), 男, 博士, 教授, 硕士生导师, CCF 会员, 主要研究方向为并 行处理器体系结构、片上网络. 
掘等. 这些应用程序由于处理的业务逻辑不同，对 资源的需求量和使用量也不相同，特别是对一些 共享资源的使用会出现相互干扰的问题 ${ }^{[2]}$. 片上网 络(network-on-chip, NoC)作为共享资源，极易出现 不同应用程序相互干扰的问题，主要体现在不同 应用程序的通信数据流会频繁地在 $\mathrm{NoC}$ 的某些节 点处相遇, 形成对路由器、传输链路等硬件资源的 竞争, 导致无法保证应用程序的网络性能 ${ }^{[3]}$. 子网 隔离方法 ${ }^{[4]}$ 将多个应用程序部署在片上系统的不 同连续子网中并行执行，并采用隔离路由算法将 不同应用程序的通信数据流限制在本子网内，从 而解决多个应用程序通信数据流在 $\mathrm{NoC}$ 中相互干 扰的问题. 为了提高系统利用率并减少碎片率, 通 常应用程序的部署子网为不规则子网 ${ }^{[5-6]}$.

维序路由(dimension order routing, DOR)算法 是最简单、功耗最低的最短路由算法, 它的路径总 是位于源节点和目标节点确定的最小象限内. 然 而，该算法只适用于规则拓扑子网，会导致更多网 络碎片，从而降低芯片利用率. 目前针对不规则拓 扑子网的隔离路由算法研究较少, 最常用的是基 于逻辑的分布式路由(logic based distributed routing, LBDR)算法 ${ }^{[7]}$, 其具有足够的可伸缩性和灵活 性, 以适应不规则网络. 然而, LBDR 算法只支持 最小路径路由, 当不规则拓扑网络不能满足最小 路径路由时, 该算法失效. Resilient-LBDR 算法 ${ }^{\left[{ }^{[8]}\right.}$ 和动态可重构路由 (dynamically reconfigurable architecture fault-tolerant routing, DRAFT)算法 ${ }^{[9]}$ 适用 于更多种不规则拓扑网络, 但是需要借助虚通道 避免死锁. 动态可扩展的分布式路由 (fault tolerant dynamic and scalable, TRACK) 算法 ${ }^{[10]}$ 采用了 d2-LBDR 算法 ${ }^{[11]}$ 的逻辑思想, 实现了 UP*/DOWN* 路由算法 ${ }^{[12]}$, 从而提高其可扩展性. 然而, 当通过 最短路径路由数据包时, TRACK 算法在网络性能 方面相比 DOR算法差. 灵活的 DOR(flexible DOR, FDOR)算法 ${ }^{[13]}$ 是典型的 (路由内含)流量隔离算法, 通过禁止特定的转向避免死锁. 该算法支持的子 网拓扑结构由 3 个 mesh 拓扑连接组成, 2 个辅 mesh 必须位于主 mesh 的两侧, 并且 3 个 mesh 拓扑的维 数必须一致. 锯齿拓扑 FDOR (FDOR jagged edges routing, FJE)算法 ${ }^{[14]}$ 拓展了 FDOR 算法, 可支持锯 齿拓扑结构, 但是其逻辑复杂。二级优先的 DOR(two priority dimension order routing, TPDOR) 算法 ${ }^{[15]}$ 拓展了 FDOR 算法, 支持 3 个 mesh 拓扑以 任意方式连接成的拓扑结构的子网, 增加了可适 用的不规则子网拓扑类型.
为了解决上述问题，本文采用区域拓扑模型 识别不规则子网, 并且结合已有研究成果的容错 奇偶 (fault-tolerant odd-even, FTOE)转向模型 ${ }^{[16]}$, 提出了一种低开销的隔离路由算法(low-cost isolation routing algorithm, LIRA). 该算法只需要在子 网边界路由器上设置一些路由规则, 继承了 DOR 算法的简单高效. 实验结果表明, LIRA 适用于多 种不规则拓扑结构的子网, 在不降低 NoC 性能的 情况下，提高了系统的利用率.

\section{1 不规则区域拓扑模型与转向模型}

\section{1 不规则区域拓扑模型}

为了将每个应用程序的通信数据流隔离到子 网内, 首先需要识别子网的不规则边界. 本文借鉴 凸故障模型 ${ }^{[17]}$ 的思想定义不规则子网的边界拓扑 模型. 根据子网边界的不规则性，不规则区域可分 为 8 类 $\left(R_{1} \sim R_{8}\right)$, 如图 1 所示.

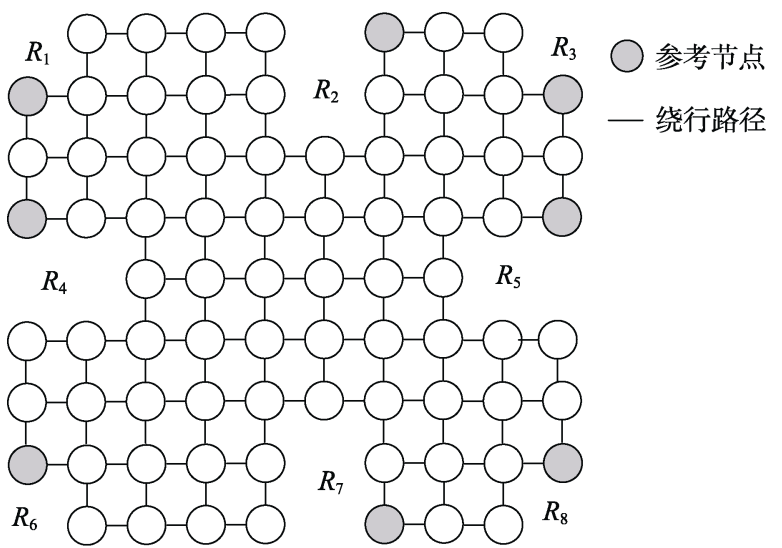

图 1 不规则区域类型

这些不规则区域周围的绕行链路称为绕行路 径, 不规则区域 $R_{2}$ 和 $R_{7}$ 的绕行路径西边界由 2 列 组成. 每条绕行路径上都有一个参考节点, 并且绕 行路径上的每个节点都记录了该参考节点的坐标 信息, 方便路由. LIRA 适用于上述 8 类不规则拓扑 区域中任意 2 种不规则区域组成的连续子网. 图 2 给出了几种典型的不规则拓扑子网应用. 图 2a 中 的不规则子网是由 $R_{4}$ 和 $R_{5}$ 组成，图 $2 \mathrm{~b}$ 中的不规则 子网是由 2 个 $R_{3}$ 组成, 图 2c 中的不规则子网是由 $R_{1}$ 和 $R_{8}$ 组成. 灰色节点为绕行路径上的节点.

\subsection{FTOE 转向模型}

本文采用适用于不规则 2D mesh 拓扑结构网 络的 FTOE 转向模型 ${ }^{[16]}$ 避免路由算法的死锁, 如 图 3 所示, 规则如下. 


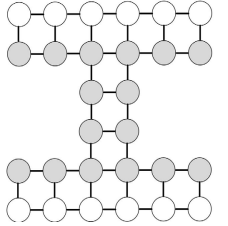

a. $R_{4}$ 和 $R_{5}$ 组合

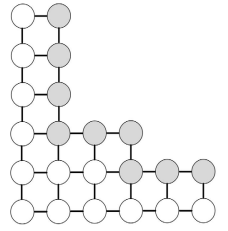

b. 2 个 $R_{3}$ 组合

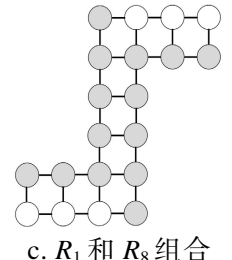

c. $R_{1}$ 和 $R_{8}$ 组合
图 2 不规则拓扑子网应用

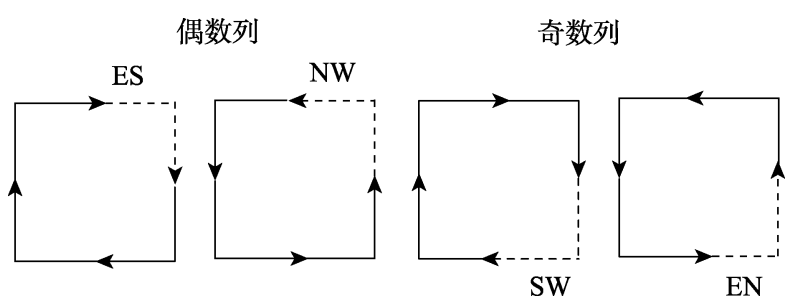

图 3 FTOE 转向模型

规则 1. 数据包在位于偶数列的节点上不允许 北西(NW)和东南(ES)转向.

规则 2. 数据包在位于奇数列的节点上不允许 南西(SW)和东北(EN)转向.

根据 FTOE 规则, 因为东南(ES)转向和南西 (SW)转向不可能出现在同一列上, 所以不能形成 顺时针最右边列. 同时, 北西 (NW)转向和东北 $(\mathrm{EN})$ 转向也不可能出现在同一列上, 所以不能形 成逆时针最右边列. 因此, FTOE 转向模型不能形 成最右边列，从而确保网络无死锁发生.

\section{2 隔离路由算法}

LIRA 由 2 步组成: 首先数据包根据 FTOE 转 向模型规则正常路由; 当数据包遇到不规则区域, 且不能正常路由时, 数据包通过绕行路径绕过不 规则区域. LIRA 支持正常路由、南北边界路由和 东西边界路由 3 种模式.

\section{1 正常路由}

根据目标节点与当前节点的相对位，如果当 前节点不在绕行路径上，或者当前节点在绕行路 径上，但与目标节点间存在最小路由路径，表明不 规则区域未影响数据包的路由.

规则 3 . 当前节点位于偶数(奇数)列且目标节 点位于源节点的西北(西南)方向时, 数据包首先朝 西传输到奇数(偶数)列, 然后根据 YX 路由规则传 输; 否则数据包根据 YX 路由规则传输.

\section{2 数据包从北边界或南边界进入绕行路径}

数据包从北边界或南边界进人绕行路径时, 不规则区域使当前节点与目标节点间不存在最小
路由路径, 则需要数据包先沿着绕行路径路由, 直 到满足最小路径路由的条件为止.

规则 4. 数据包从北边界进人绕行路径, 并且 目标节点位于参考节点的东边 (西边), 则数据包顺 时针(逆时针)沿着绕行路径路由, 如图 4 所示, 其 中 $C$ 和 $R$ 分别表示当前节点和参考节点, 下文中变 量含义相同.

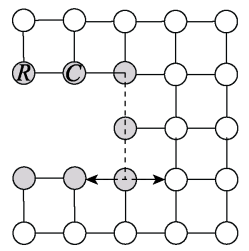

a. 进人区域 $R_{4}$

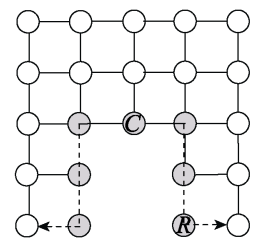

b. 进人区域 $R_{7}$

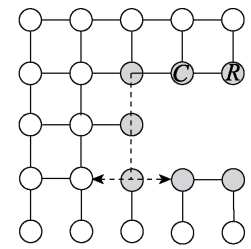

c. 进人区域 $R_{5}$
图 4 数据包从北边界进人绕行路径

规则 5. 数据包从南边界进人绕行路径, 并且 目标节点位于参考节点的东边 (西边), 则数据包逆 时针(顺时针)沿着绕行路径路由, 如图 5 所示.

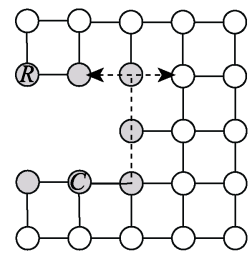

a. 进人区域 $R_{4}$

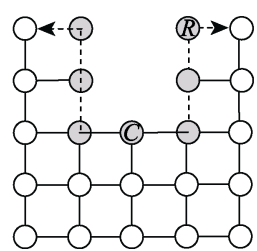

b. 进人区域 $R_{2}$

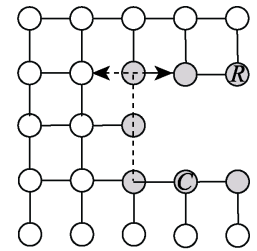

c. 进人区域 $R_{5}$
图 5 数据包从南边界进人绕行路径

\section{3 数据包从东边界或西边界进入绕行路径}

当数据包从西边界进人绕行路径, 且目标节 点位于不规则区域的东边, 这样在绕行路径的西 边界会引人被禁止的转向. 为了避免引人被禁止 的转向, 绕行路径的西边界由 2 列组成.

规则 6. 数据包从西边界进人绕行路径, 并且 目标节点位于参考节点的南边 (北边), 则数据包首 先传输到西边界的奇数(偶数) 列, 然后顺时针(逆 时针)沿着绕行路径路由, 如图 6 所示. 其中, $O$ 和 $E$ 分别表示当前网络的奇数列和偶数列.

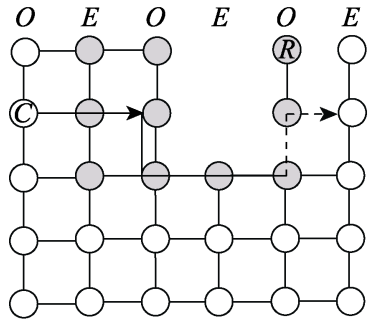

a. 进人区域 $R_{2}$

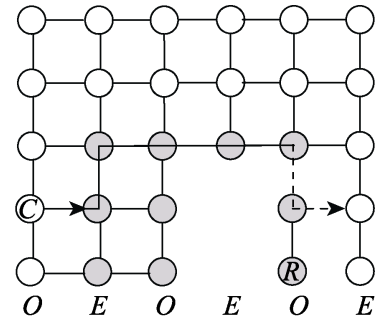

b. 进人区域 $R_{7}$
图 6 数据包从西边界进人绕行路径 
规则 7. 数据包从东边界进人绕行路径, 并且 目标节点位于参考节点的南边(北边), 数据包逆时 针(顺时针)沿着绕行路径路由, 如图 7 所示.

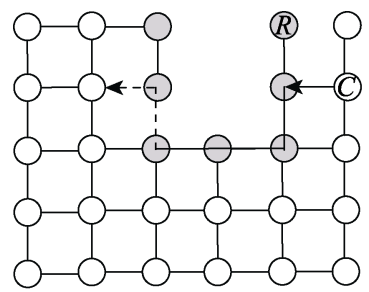

a. 进人区域 $R_{2}$

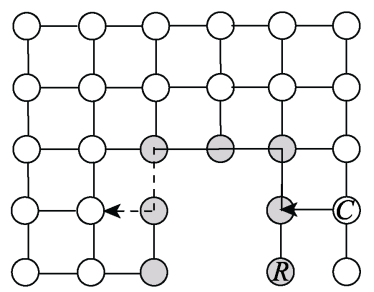

b. 进人区域 $R_{7}$
图 7 数据包从东边界进人绕行路径

\section{3 无死锁和无活锁证明}

Dally 等 ${ }^{[17]}$ 提出: 如果由某个路由算法构造的 通道依赖图(channel dependency graph, CDG)不存 在封闭环路, 那么该路由算法无死锁. 付斌章等 ${ }^{[18]}$ 证明了如果所有环路都没有形成最右边列, 那么 不能形成封闭 CDG 环路.

引理 1. 数据包未遇到子网的不规则边界时路 由, 不能形成封闭 CDG 环路.

证明. 未进入绕行路径时, 数据包根据规则 3 传输, 因此数据包传输不能形成最右边列, 从而避 免了封闭 CDG 环路的形成.

证毕.

引理 2. 数据包从南边界或北边界进人绕行路 径，不能形成封闭 CDG 环路.

证明. 数据包在抵达绕行路径的南边或北边 之前, 根据规则 3 传输, 使偶数列避免了 $\mathrm{NW}$ 转向、 奇数列避免了 SW 转向; 因此数据包传输不能形成 最右边列，避免了封闭 CDG 环路的形成. 证毕.

引理 3. 数据包从西边界进人绕行路径, 不能 形成封闭 CDG 环路.

证明. 当数据包抵达绕行路径的西边界列时, 根据规则 6 传输, 2 个西边界列为数据包提供足够的 灵活度来应对所有的转向, 使偶数列避免了 ES 转 向, 并且奇数列避免了 EN 转向; 因此数据包传输 不能形成最右边列, 避免了封闭 CDG 环路的形成.

证毕.

引理 4. 数据包从东边界进人绕行路径, 不能 形成封闭 CDG 环路.

证明. 当数据包抵达绕行路径的东边界列时, 根据规则 7 传输, 不能出现最右边列的转向, 因此 数据包传输不能形成封闭 CDG 环路.

证毕.

引理 5. 数据包沿着绕行路径传输, 不能形成
封闭 CDG 环路.

证明. 当数据包沿着绕行路径传输时, 可能会 形成最右边列. 根据第 1.1 节中不规则区域拓扑模 型, 可以看出每个绕行路径至少缺少一条边界, 因 此数据包沿着绕行路径传输, 不能形成封闭 CDG 环路.

证毕.

结论 1. LIRA 无死锁.

证明.

(1) 根据引理 1, 数据包未进人绕行路径时, 不能形成封闭 CDG 环路.

（2）根据引理 2 引理 4, 数据包抵达绕行路径 上的任意节点, 都不能形成封闭 CDG 环路. 根据 引理 5, 数据包沿着绕行路径传输, 不能形成封闭 CDG 环路, 因此 LIRA 无死锁.

证毕.

结论 2. LIRA 无活锁.

证明. 如果源节点和目标节点之间不存在不 规则区域, LIRA 是最短路径路由算法; 否则, 数据 包沿着绕行路径传输, 直到绕到与目标节点同列 或同行时被终止. 因此 LIRA 无活锁。证毕.

\section{4 仿真分析}

实验对模拟器 Booksim 2.0 $0^{[19]}$ 进行修改, 以模 拟隔离路由算法. 同时将 Orion 2.0 模型集成到 Booksim 2.0 中进行功耗仿真. 仿真分为系统利用 率分析、性能分析和功耗分析 3 部分. 其中性能分 析又包括真实应用踪迹测试和合成负载通信测试.

\section{1 系统利用率分析}

一个较高的系统利用率意味着有更多的应用 程序被运行在众核处理器上. 本文采用覆盖率作 为系统利用率的度量指标. 它表示路由算法所支 持的不规则拓扑子网数量占所有不规则拓扑子网 的百分比. 覆盖率越高, 说明可同时运行在众核处 理器上的应用程序个数越多, 也即意味着较高的 系统利用率.

不规则拓扑子网可以在规则 mesh 网络中随机 禁用某几个节点而生成, 部分节点被禁用后会形 成一组不同大小的分区，使每个节点都是分区的 一部分. 然后检验路由算法是否满足该不规则拓: 扑子网的路由. 评估采用 1 个 $4 \times 42 \mathrm{D}$ mesh 网络和 1 个 $8 \times 8$ 2D mesh 网络. 对于每个拓扑网络进行 30000 次实验, 每次实验都包含一组随机的 1 5个 禁用节点(禁用节点数随机均匀分布).

本文对 LBDR-XY 算法、TPDOR 算法和 LIRA 
进行覆盖率仿真, 仿真结果如图 8 所示. 从图 8 中 可以看出, LIRA 覆盖率最高, 比 LBDR-XY 算法和 TPDOR算法分别提高了 $57.03 \%$ 和 $25.09 \%$. 这是因 为 LIRA 可以容忍图 1 中 8 类不规则拓扑区域中任 意 2 种不规则区域组成的连续子网. LBDR-XY 算 法的覆盖率最低, 因为它只支持满足最小路径路 由的不规则拓扑网络.

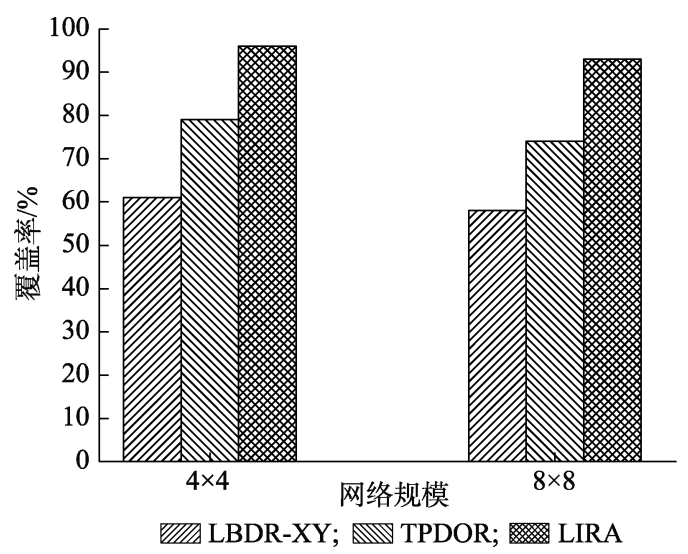

图 8 覆盖率仿真

\section{2 系统性能分析}

\subsection{1 真实应用踪迹测试}

实验采用 2 个模拟器评估全系统性能. 首先采 用全系统仿真器 $M 5^{[20]}$, 具体配置如表 1 所示. 在 仿真器上运行 PARSEC 测试集, 并使用 Netrace ${ }^{[21]}$ 工具收集内存之间的访问关系; 然后对其进行编 码生成一个编码文件, 最后根据编码文件内容产 生数据包, 并将这些数据包注人到 Booksim2.0 仿 真器中评估路由算法的网络延时.

\section{表 1 全系统模拟参数}

\begin{tabular}{ll}
\hline \multicolumn{1}{c}{ 参数名 } & \multicolumn{1}{c}{ 参数值 } \\
\hline 核数 & 64 \\
$L_{1}$ Cache & Private, 4-way, 32 KB \\
$L_{2}$ Cache & Public, 8-way, 16 MB \\
一致性协议 & MOESI_CMP_directory \\
网络拓扑 & $8 \times 8$ 2D Mesh \\
\hline
\end{tabular}

图 9 给出了 LBDR-XY 算法和 LIRA 在 PARSEC 程序下相对于 TPDOR 算法的全系统延时对比. 4 个应用程序被划分成 2 种类型：低网络负载和高网 络负载. 全系统性能取决于每个程序产生的负载 和流量模式, 具有较高网络负载的应用程序能够 从优化的路由算法上获得性能提升. 例如, 在具 有高网络负载的 canneal 和 fluidanimate 程序中, 这 2 种算法的性能提升相对比较小. 在具有低网络
负载的 x264 和 blackscholes 程序中, LBDR-XY 算 法和 LIRA 的算法性能提升较大. 比起 TPDOR 算 法, LBDR-XY 算法和 LIRA 的网络延时分别减小 了 $2.29 \%$ 和 $2.56 \%$.

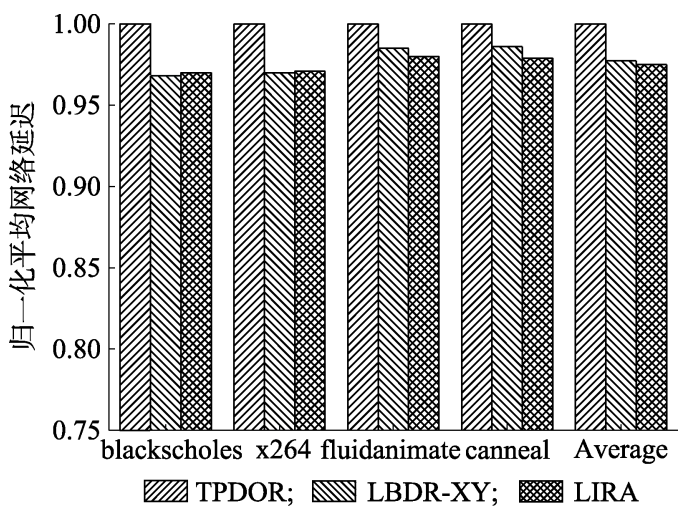

图 9 PARSEC 测试集下的网络延时对比

\subsection{2 合成负载通信模式测试}

合成负载通信模式仿真中，每个输人通道的 缓存大小为 8 个 flit, 数据包长度在 2 5 个 flit 之间 均匀分布. 除了 LIRA, 本文还实现了 LBDR-XY 算法(采用 $X Y$ 规则的 $\mathrm{LBDR}^{[7]}$ 算法)和 $\mathrm{TPDOR}^{[15]}$ 算法与之进行比较.

在 8×8 2D mesh 网络实验中, 使用了 2 种子网 配置方案: 规则子网配置和不规则子网配置, 如图 10 所示. TPDOR 算法只支持 3 个 mesh 拓扑连接成 的拓扑结构, 因此不规则子网配置为 3 个 mesh 拓 扑组合. 每个子网就像一个独立的网络, 都使用相 同的路由算法, 并独立注人数据包和统计其延迟.

为了评估算法的性能, 实验使用了 3 种流量模 式 uniform, transpose 和 hotspot. 其中, hotspot 模式 是在 uniform 模式下将 $5 \%$ 的数据包发送到某个热 点节点. 在图 10 的各个子网中, 实验只关注子网 SN1 的性能. 测试子网 SN1 在不同流量模式下的 性能, 子网 SN2, SN3 和 SN4 传输 uniform 模式, 注人率为 0.04 flits/cycle/node. LBDR-XY 算法不支 持 SN1 不规则拓扑子网, 因此在不规则区域中只 仿真 LIRA 和 TPDOR 算法.

从图 11a 中可以看出, 在规则子网中, TPDOR 和 LIRA 的算法性能稍差于 LBDR-XY 算法, 这主 要是因为 LBDR-XY 算法采用 $X Y$ 算法传输数据 包, 它是确定性路由算法, 且其在 uniform 模式下 具有高吞吐率及低延时性能. 从图 12a 中可以看 出, 在不规则子网中, uniform 模式下 TPDOR 和 LIRA 具有相似的算法性能. 


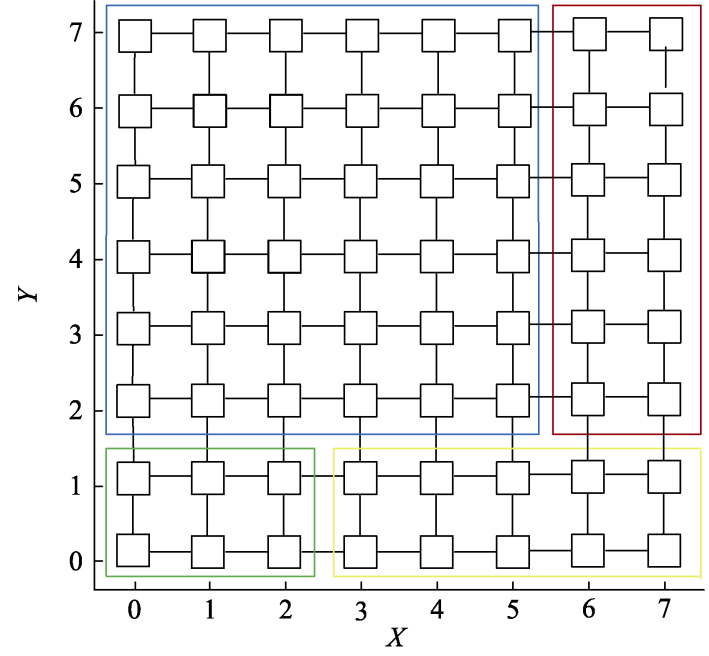

$\square \mathrm{SN} 1 ; \square \mathrm{SN} 2 ; \square \mathrm{SN} 3 ; \square \mathrm{SN} 4$ a. 规则子网

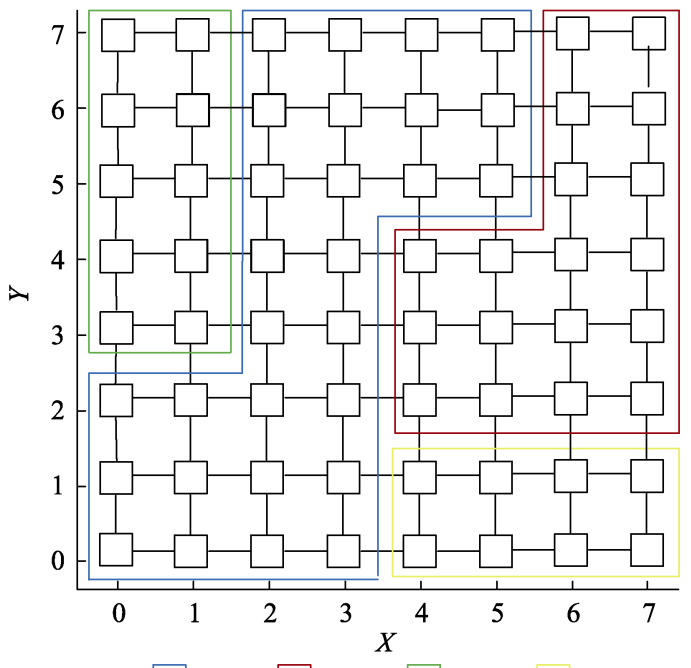

$\square \mathrm{SN} 1 ; \square \mathrm{SN} 2 ; \square \mathrm{SN} 3 ; \square \mathrm{SN} 4$ b. 不规则子网

图 $108 \times 8$ 网络子网配置

在 transpose 模式下, 网络经常产生很多具有 相同目标节点的数据包，即这些数据包采用相同 的路径到达目标节点, 这样容易发生网络负载不 均衡, 随着包注人率的增加, 网络很快进入拥塞. 从图 $11 \mathrm{~b}$ 中可以看出, 在规则子网中, 3 种路由算 法具有相似的性能. 从图 $12 \mathrm{~b}$ 中可以看出, 在不规 则子网中，比起 TPDOR 算法, LIRA 的饱和吞吐率 增加了 $3.7 \%$, 这主要是因为 transpose 模式容易发 生网络负载不均衡, FTOE 转向模型具有流量均衡 能力, 从而使 LIRA 的算法性能优于 TPDOR

hotspot 模式是在 uniform 模式下将 5\%的数据 包发送到热点节点 $(3,5)$. 从图 11c 中可以看出, 在 规则子网中，这 3 种隔离路由算法具有相似的性 能. 从图 12c 中可以看出, 在不规则子网中, 比起
TPDOR 算法, LIRA 的饱和吞吐率增加了 $4.2 \%$. 这 主要因为 hotspot 模式使网络拥塞频繁发生, FTOE 转向模型具有流量均衡能力, 从而使 LIRA 的算法 性能优于 TPDOR.

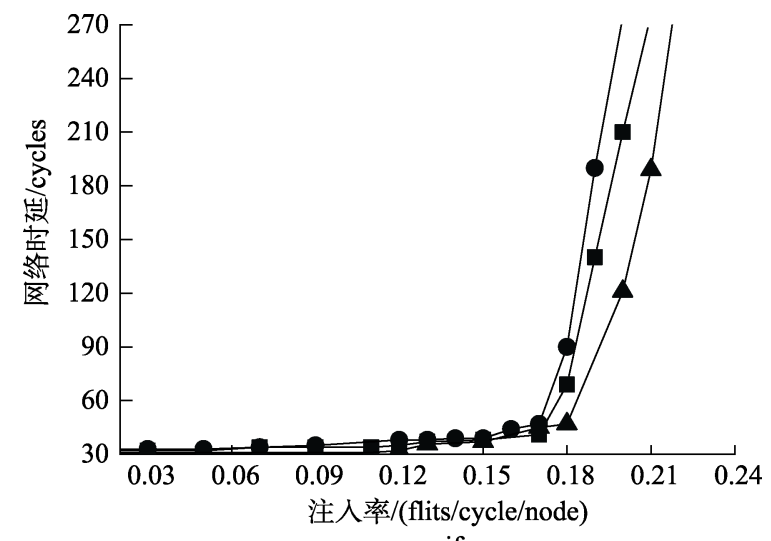
a. uniform
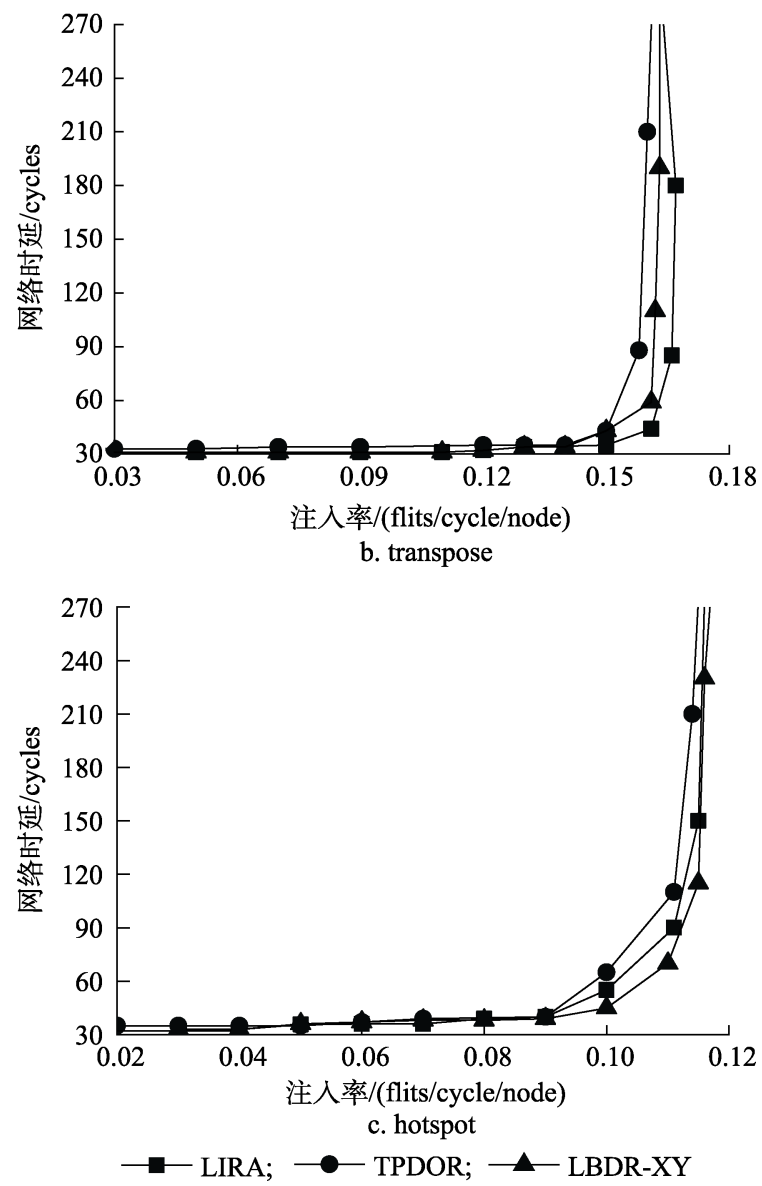

图 11 路由算法在 $8 \times 8$ 网络中规则 拓扑子网上的性能

扩展网络大小到 16×16 2D mesh 网络, 不规则 子网配置如图 13 所示. 在图 14 的各个子网中, 实 验只关注子网 SN1 的性能. 测试子网 SN1 在不同 流量模式下的性能, 子网 SN2, SN3 和 SN4 传输 uniform 模式, 注人率为 0.02 flits/cycle/node. 

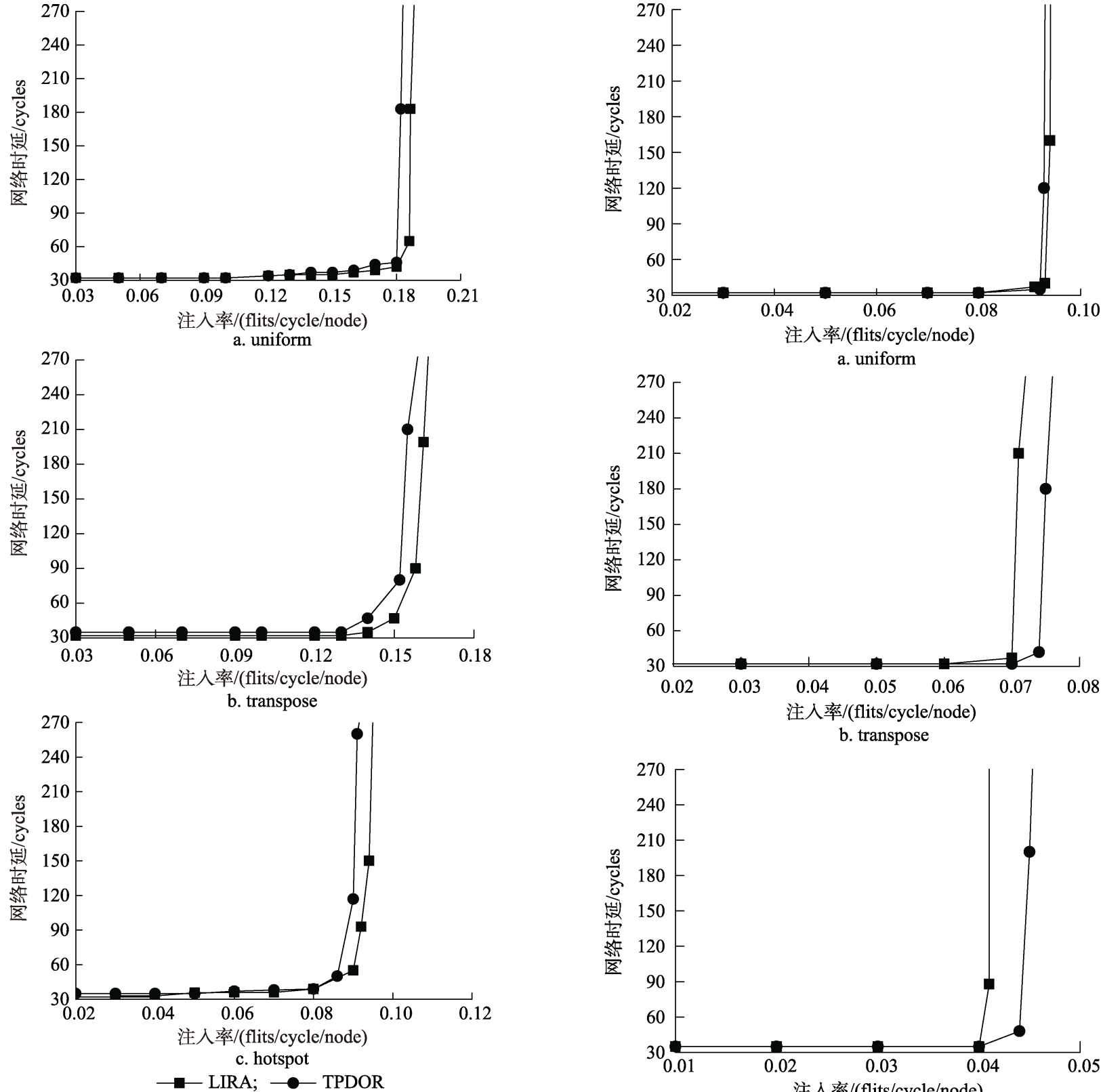

图 12 路由算法在 $8 \times 8$ 网络不规则拓扑子网上的性能
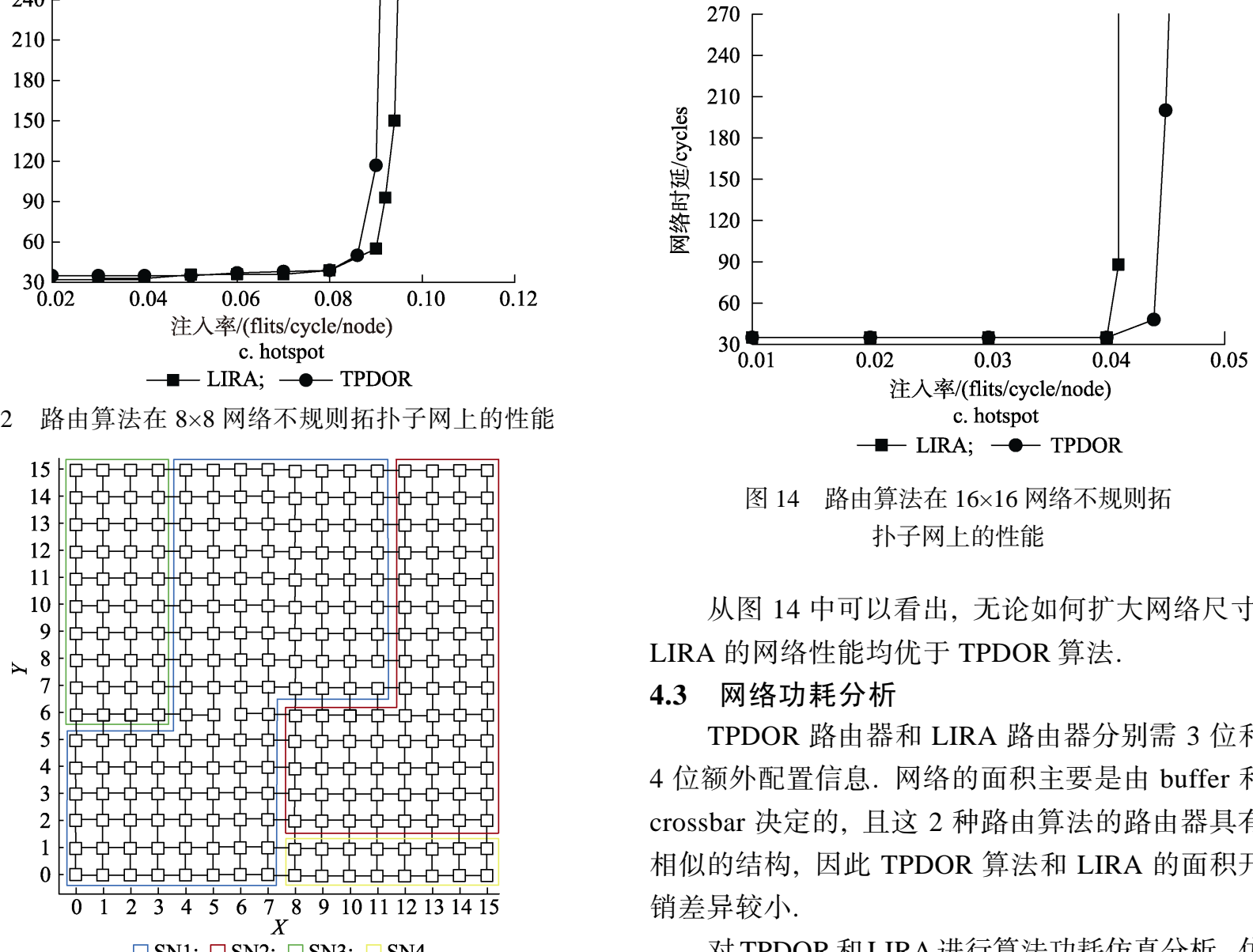

图 14 路由算法在 $16 \times 16$ 网络不规则拓 扑子网上的性能

从图 14 中可以看出, 无论如何扩大网络尺寸, LIRA 的网络性能均优于 TPDOR 算法.

\section{3 网络功耗分析}

TPDOR 路由器和 LIRA 路由器分别需 3 位和 4 位额外配置信息. 网络的面积主要是由 buffer 和 crossbar 决定的, 且这 2 种路由算法的路由器具有 相似的结构, 因此 TPDOR 算法和 LIRA 的面积开 销差异较小.

对 TPDOR 和 LIRA 进行算法功耗仿真分析. 仿 图 $1316 \times 16$ 网络不规则子网配置 真采用 ITRS $65 \mathrm{~nm}$ 工艺参数, 工作频率为 $500 \mathrm{MHz}$, 
工作电压为 $0.8 \mathrm{~V}$, 链路宽度为 64 bit. 采用图 10b 中 的不规则子网配置, 子网 SN2, SN3 和 SN4 传输 uniform 模式, 注人率为 0.04 flits/cycle/node. 子网 SN1 在 transpose 通信模式, 分别采用中等注人率 (0.14 flits/cycle/node) 和高注人率 (0.155 flits/cycle/ node). 表 2 给出这 2 种路由算法的功耗. 在 0.155 flits/cycle/node 注人率下, 比起 LIRA, TPDOR 算法 的功耗增加了 $1.2 \%$. 这主要是因为 LIRA 采用的 FTOE 转向模型具有流量均衡能力.

表 22 种算法在 transpose 模式不同注入率下的功耗

\begin{tabular}{ccc}
\hline 注人率 & TPDOR 算法/W & LIRA/W \\
\hline 0.140 & 1.7412 & 1.7223 \\
0.155 & 1.9320 & 1.9089 \\
\hline
\end{tabular}

\section{4 仿真结果讨论}

根据系统利用率分析可以看出, 与 LBDR-XY 和 TPDOR 算法相比, LIRA 的利用率提高较多. 根 据网络性能分析可以看出, 在规则子网中, LBDR, LIRA 和 TPDOR 具有相似的算法性能; 在不规则 子网中, 由于 FTOE 转向模型具有流量均衡能力, LIRA 的网络性能稍好于 TPDOR 算法. 根据功耗 分析可以看出, 与 LIRA 相比, TPDOR 算法的功耗 增加了 $1.2 \%$.

综上所述, 在系统网络性能和功耗方面, LIRA 的优势不是很明显. 但在系统利用率方面, 其只支持满足最小路径路由的不规则子网; TPDOR 算法只支持 3 个 mesh 拓扑以任意方式连 接成的拓扑结构的子网. LIRA 不仅适用于 LBDR 和 TPDOR 算法所支持的所有不规则子网，还支持 第 1.1 节中 8 类不规则区域中任意 2 种不规则区域 组成的连续拓扑子网, 从而提高了系统的利用率.

\section{5 结 语}

本文针对片上众核处理器中, 大量应用程序 并发执行时，应用程序所部署的连续区域间通信 数据流干扰的问题, 提出了不规则区域拓扑结构 模型用于识别不规则子网的边界, 再设计了一种 低开销的无虚通道隔离路由算法(LIRA). 该算法 采用 DOR 算法路由, 一旦数据包遇到不规则区域 破坏了最小路由路径, 则改变路由策略, 直到满足 最小路径路由的条件. 该算法既继承了 DOR 算法 的简单高效，又能适用于多种不规则拓扑结构的 子网, 从而提高了系统的利用率. 为了避免引人额 外的面积开销, 本文采用 FTOE 转向模型来避免死
锁，并且理论证明了 LIRA 的无死锁和无活锁的特

性. 实验结果表明, LIRA 在没有提高网络性能和 功耗开销的前提下，提高了系统利用率.

\section{参考文献(References):}

[1] Hesham S, Goehringer D, Abd EI Ghany M A. HPPT-NoC: a dark-silicon inspired hierarchical TDM NoC with efficient power-performance trading[J]. IEEE Transactions on Parallel and Distributed Systems, 2020, 31(3): 675-694

[2] Lu Hang. Performance isolation and power management approaches for NoC-based manycores[D]. Beijing: University of Chinese Academy of Sciences Computer System and Architecture, 2015(in Chinese)

(路航. 片上网络众核处理器的性能隔离与功耗管理方法研 究[D]. 北京: 中国科学院大学计算机系统结构, 2015)

[3] Sadeghi M S, Sarmadi S B, Hessabi S. Toward on-chip network security using runtime isolation mapping[J]. ACM Transactions on Architecture and Code Optimization, 2019, 16(3): Article No.28

[4] Chatterjee N, Paul S, Mukherjee P, et al. Deadline and energy aware dynamic task mapping and scheduling for network-on-chip based multi-core platform[J]. Journal of Systems Architecture, 2017, 74: 61-77

[5] Pranzo M, Mazumdar S. An analytical model for thread-core mapping for tiled CMPs[J]. Performance Evaluation, 2019, 134: 102003

[6] Flich J, Duato J. Logic-based distributed routing for NoCs[J]. IEEE Computer Architecture Letters, 2008, 7(1): 13-16

[7] Bishnoi R, Laxmi V, Gaur M S, et al. Resilient routing implementation in 2D mesh NoC[J]. Microelectronics Reliability, 2016, 56: 189-201

[8] Bahrebar P, Jalalvand A, Stroobandt D. Dynamically reconfigurable architecture for fault-tolerant 2D networks-on-chip[C] //Proceedings of the 26th International Conference on Computer Communication and Networks. Los Alamitos: IEEE Computer Society Press, 2017: 1-7

[9] Jain A, Laxmi V, Tripathi M, et al. TRACK: an algorithm for fault-tolerant, dynamic and scalable 2D mesh network-on-chip routing reconfiguration[J]. Integration, 2020, 72: 92-110

[10] Bishnoi R, Laxmi V, Gaur M S, et al. d²-LBDR: distance-driven routing to handle permanent failures in 2D mesh NoCs[C] //Proceedings of the Design, Automation \& Test in Europe Conference \& Exhibition. Los Alamitos: IEEE Computer Society Press, 2015: 800-805

[11] Schroeder M D, Birrell A D, Burrows M, et al. Autonet: a high-speed, self-configuring local area network using point-to-point links[J]. IEEE Journal on Selected Areas in Communications, 1991, 9(8): 1318-1335

[12] Skeie T, Sem-Jacobsen F O, Rodrigo S, et al. Flexible DOR routing for virtualization of multicore chips[C] //Proceedings of IEEE International Symposium on System-on-Chip. Los Alamitos: IEEE Computer Society Press, 2009: 73-76

[13] Sem-Jacobsen F O, Rodrigo S, Skeie T, et al. An efficient, low-cost routing framework for convex Mesh partitions to 
support virtualization[J]. ACM Transactions on Embedded Compuing Systems, 2013, 12(4): Article No.107

[14] Sun Guang, Zhang Yuanyuan, Li Yong, et al. Two priority dimension order routing algorithm for virtualization of a network on chip design[J]. Journal of Tsinghua University: Science and Technology, 2011, 51(3): 416-419(in Chinese)

(孙光, 张媛媛, 李勇, 等. 基于虚拟化片上网络的二级优先 维序路由算法[J]. 清华大学学报: 自然科学版, 2011, 51(3): 416-419)

[15] Xie R L, Cai J P, Xin X. Simple fault-tolerant method to balance load in network-on-chip[J]. Electronics Letters, 2016, 52(10): 814-816

[16] Boppana R V, Chalasani S. Fault-tolerant routing with non-adaptive wormhole algorithms in mesh networks[C] //Proceedings of the ACM/IEEE Conference on Supercomputing. Los Alamitos: IEEE Computer Society Press, 1994: 693-702

[17] Dally W J, Seitz C L. Deadlock-free message routing in multi- processor interconnection networks[J]. IEEE Transactions on Computers, 1987, 36(5): 547-553

[18] Fu Binzhang, Han Yinhe, Li Huawei, et al. Building resilient NoC with a reconfigurable routing algorithm[J]. Journal of Computer-Aided Design \& Computer Graphics, 2011, 23(3): 448-455(in Chinese)

(付斌章, 韩银和, 李华伟, 等. 面向高可靠片上网络通信的 可重构路由算法[J]. 计算机辅助设计与图形学学报, 2011, 23(3): 448-455)

[19] Dally W J, Towles B. Principles and practices of interconnection networks[M]. San Francisco: Morgan Kaufmann, 2004

[20] Ma S, Jerger N E, Wang Z Y, et al. Holistic routing algorithm design to support workload consolidation in NoCs[J]. IEEE Transactions on Computers, 2014, 63(3): 529-542

[21] Hestness J, Grot B, Keckler S W. Netrace: dependency-driven trace-based network-on-chip simulation[C] //Proceedings of the 3rd International Workshop on Network on Chip Architectures. New York: ACM Press, 2010: 31-36 